\title{
Future of CAE and Implication on Engineering Education
}

\author{
Seung-Hyun Yoo ${ }^{1}$, Eung-Jun Park ${ }^{2}$, Jae-Sil Lee ${ }^{2}$, Joon-Ho Song ${ }^{2}$, Dae-Jin \\ $\mathrm{Oh}^{2}$, Woong-Rak Chung ${ }^{2}$, Yeongtae Lee ${ }^{2}$, and Dhaneshwar Mishra ${ }^{2}$ \\ 1 Department of Aerospace Engineering, University of Maryland, College \\ Park, Maryland, USA, On leave from Department of Mechanical \\ Engineering, Ajou University, Suwon, Korea ryseung@gmail.com \\ 2 Department of Mechanical Engineering, Ajou University, Suwon, Korea \\ WWW home page: http://www.ajou.ac.kr/ sml
}

\begin{abstract}
In this paper, the trend of FEM which is one of core tools of CAE and the future of CAE are examined in terms of TRIZ concept. This observation leads to the necessity of engineering creativity. This paper reports our successful experiences of implementing systematic innovation tools into engineering design classes. Also potential revolutionary change of engineering education is hinted by extending the accomplishments of TRIZ. It can innovate the current engineering curricula and an on-going study of classifying the engineering activities is introduced.
\end{abstract}

\section{Introduction}

Every modern organizations needs innovation tools in order to adapt in rapidly changing environments, especially modern companies [1-3]. Radical innovations require three elements, namely, they should be new to market, $5 \sim 10$ times more productive and show 30 50\% cost reduction. It is generally accepted that current curricula of engineering education and engineering practices cannot meet these requirements. We have been trying to find a systematic tool for innovation, creative design, and inventions using CAE (Computer Aided Engineering) tools such as FEM (Finite Element Method) as the authors have been teaching structural mechanics courses in conjunction with engineering design. Current curricula of mechanical engineering consists of basic sciences such as mathematics, physics, chemistry, and biology (this course was added recently to expand current mechanical engineering areas) and mechanics courses such as statics, dynamics, solid and fluid mechanics, thermodynamics and design practices from machine elements design to system design. Those core disciplines are called engineering sciences and their primary goals are to understand the materials and systems in order to simulate and predict the 
behaviors. As our students are going out and will spend several decades in industry, our concern is about the future of $\mathrm{CAE}$ and how to prepare them to retain competency throughout their whole careers. So this paper will report on the history of FEM and a prediction for the future of CAE. Naturally, we will discuss the importance of systematic innovation tools and our experiences with implementing this into engineering design class and feasibility of engineering curricula reformation are introduced. So the first part will examine current practice of CAE and the needs of systematic innovation tools. The second part describes roles of CAI in engineering design classes.

\section{Advancement of CAE}

\subsection{History of FEM}

Considerable achievements have been made to avoid catastrophic failures, although there have been some exceptions such as the 2003 Columbia accident [4]. And one of the achievements of CAE is to make robust estimation of durability. CAE is critical element in VPD (Virtual Produce Development) in conjunction with current digital enterprise including ERP, PLM, and SCM. The core of current CAE practice is FEM as shown in Fig. 1.

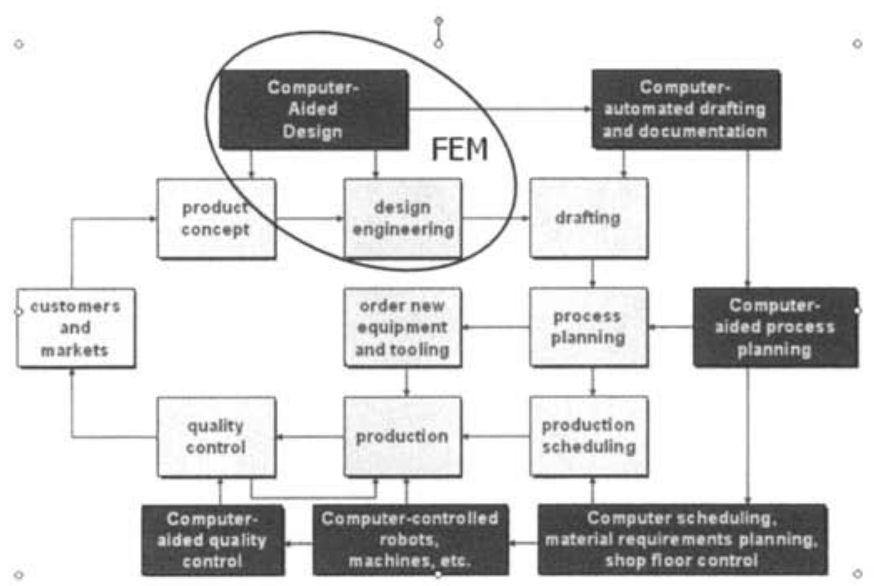

Fig. 1. FEM in CAE processes

The first part of this section investigates the evolution of FEM. Simulations of product behavior are necessary for good design. Mostly the governing equations are given as differential equations and the final problems become BVP (Boundary Value Problems) or IVP (Initial Value Problems). Analytical solutions can be obtained for simple geometries with simple conditions and exhaustive solutions are documented 
for many cases. Many eminent applied mathematicians and engineers contributed on the exact solutions, but most real problems do not allow analytical solutions. Therefore we should resort to numerical methods such as FDM (Finite Difference Method) and FEM. The discretization methods are developed for this purpose and FDM has longer history than FEM in this sense. But FEM plays more important role as the de facto standard method nowadays. FEM uses 'Divide and Conquer' concept and discretizing techniques. The FEM has developed into current form by combining variational and weighted residual method $[5,6]$. In the beginning, continuous trial functions were applied to discrete structures such as trusses and extended to continuum objects later. The modern concept of FEM was initiated in the 1950's while the implementation was done in the 1960's. Core concepts were ripe during the 1970's and extended to every possible problem after digital computers were introduced and increased their capacitances. Naturally, FEM was applied to structural analysis first and the three dimensional formulation was established. Ironically the solution process is easier for $3 \mathrm{D}$ problem than $2 \mathrm{D}$ problems such as plates and shells. The numerical techniques for these thin structures are still hot research topics. Next FEM solved more PDE's which govern stress and thermal problems. It is quite surprising that the evolution of FEM followed exactly the technology evolution pattern suggested by TRIZ. TRIZ is not explained here and readers can find information about TRIZ easily [7]. This pattern is summarized as MeThAChEM - which means Mechanical, Thermal, Acoustical, Chemical, Electrical, Magnetical and Electromagnetical. Those solutions are the first phase of MeThAChEM, namely mechanical problems. Usually PDE's are categorized into elliptic, parabolic, and hyperbolic types. The stress analysis problems are elliptic and the thermal problems are parabolic. The third is hyperbolic problems and the representative problem is a wave propagation problem. Mathematical solutions had long histories for this type of problem, but practical application of FEM to this kind appeared around 1970's which was much later than the former two types [8]. The solutions of wave phenomena are A (Acoustical) in MeThAChEM. Next applications are on chemical problems where the mass transfer is the most important distinction between mechanical and chemical analysis. The practical finite element solutions appeared on mid 1980's long after the mechanical solutions were obtained. Recently electro-magnetic problems have been analyzed by finite element method. It is wondrous to the author that the development of finite element and application procedures followed the basic concepts of TRIZ even though it had been developed independently for long time. The FEM started from dealing with matters in terms of continuum but now it is used to analyze field problems. Microscopically FEM was developed to handle discrete structures such as trusses. It was extended to deal with continuum later. It is ironic that FEM is now struggling to successfully combine with quantum mechanics which has a discontinuous nature. Those researches are being done in the realm of meso-mechanics. Although there is much literatures on the history of FEM, it is the author's opinion that this is the first attempt to combine FEM and TRIZ. 


\subsection{Future of CAE}

Naturally the next question is to predict the future of CAE where FEM represents a core element. This general pattern is also quite similar to the direction of ideality of TRIZ, which means the output from input goes infinity as the system evolves. CAE will also follow the incremental pattern of ideality and the future can be described as follows. The hardwares of computing will give following potentials at around 2020 . Typical consumer computers will have multiprocessors, 3 Tera $\mathrm{Hz} \mathrm{cpu,} 1 / 2$ TB of RAM and $50 \mathrm{~TB}$ of disk space. And the sensors will be cheap and ubiquitous [9]. So the engineering analysts used to fulfill the role of programmers and could now be categorized as analysts should be users and operators of these facilities in the future. For the software side, the HCI (Human computer interface) will improve consistently until we reach the stage where every engineer can perform any simulation he wants to do without difficulty. In our laboratory, we are using ABAQUS for FE analysis and HyperMesh for pre-post processing. Both analysis and pre-post processing softwares are improving very much for every version upgrade. Besides, COMSOL claims it can provide multi-physics capabilities inherent in its software. Also many symbolic computation softwares enable scientists and engineers to simulate whatever they want to do. They include MATHEMATICA, MACSYMA, MAPLE and MATLAB. Recent report on the research direction in computational mechanics, where the main tool is finite element, selected the following items as the main themes to dig into in the future: Virtual design, multi-scale modeling, adaptivity, very large-scale parallel computing, biomedical engineering and controlling uncertainty [10]. One of the important problems is to consider uncertainty in design parameters. There are 3 kinds of uncertainties, namely material property variability (Fatigue), manufactured shape variability (Tolerance) and design loads variability (Environmental). Almost all deterministic problems have been solved and the probabilistic problems are now main research topics. When we follow the trend of the problem types and computational power, the evolution of FEM may be summarized as follows.

- 1970's

- Linear statics, Buckling, Modal analysis

- 5,000 grids, 3,000 elements, 25,000 DOF

- 2,000 CPU sec, 1 Elapsed hr, 8MB memory, $300 \mathrm{MB}$ disk

- 1980 's

- Nonlinear, Dynamics, Superelements, Direct methods

- 50,000 Grids, 60,000 Elements, 250,000 DOF

- 2,000 CPU sec, 1 Elapsed hr, 480 MB memory, 1GB disk

- 1990's

- Adaptivitiy, Optimization, Coupled analysis

- 150,000 Grids, 200,000 Elements, 1.5M DOF,

- $\quad 6,500 \mathrm{CPU}$ sec, 2 Elapsed hr, 1GB memory, 100GB disk

- 2000's

- Probabilistic, CAD integration, AI

- No limit (As hardware allows) 
The relationship between TRIZ and CAE or FEM is investigated from the history of FEM. The first applications of primitive form of FEM were on discrete structures and extended to continuum problems later. This process can be considered as an example of 6th rule among evolution patterns of technical systems in TRIZ, increasing complexity, and 4th rule, increasing dynamism. And the development of modern FEM follows exactly the same patterns of MeThAChEM as discussed. Although the origin and main concepts are mathematical and computational, the patterns of evolution of the FEM are amazingly same as those used by TRIZ. So the future of $\mathrm{CAE}$ or FEM can be predicted by extending the line of evolution pattern. Certainly CAE will advance in the direction of increasing ideality. From the research items in the report [10], the patterns of TRIZ can be matched as follows.

Virtual design : As the system evolves increasing ideality, everything could be designed using field concept instead of substances. So the CAE systems will be virtual.

Multi-scale modeling : So far FEM is used for continuum level simulation. But the capability of FEM will be on meso and quantum level. Naturally multi-scale modeling will be an essential feature of future FEM. This is exactly same as $7^{\text {th }}$ rule of the TRIZ pattern, transition from macro to micro.

Adaptivity : This can be matched with $2^{\text {nd }}$ rule, increasing ideality, $4^{\text {th }}$ rule, increasing controllability and $8^{\text {th }}$ rule, increasing automation.

Very large-scale parallel computing : There are two ways to handle big computational tasks. One is to develop a faster chip and build so-called supercomputers. But this trend has inherent limitation and increases the expenditure very much. The other approach is to use parallel systems. This method is good to make super computing power using normal pc's. Also it is well known that new projects are developed to use idle computers to solve big problems which can not be solved by one super computer. World Community Grid is one example of that kind of effort [11]. This matches well with the main concept of TRIZ, which emphasizes 'use of every possible resource'.

Biomedical engineering: The other big area for future engineering application is $\mathrm{BME}$ (Biomedical engineering). Recently bionics and TRIZ were combined together for creative design methodology. One of the future challenges of FEM is also for exploration of living systems.

Controlling uncertainty : This can be matched with $8^{\text {th }}$ rule, decreasing human involvement or increasing automation.

When we apply IFR(Ideal Final Results) of TRIZ to the computer systems, the final computing practices will be done without expensive hardwares or system softwares. Every engineer should be able to do realistic simulations without profound knowledge on finite element theory or computing systems and programming languages. My graduate students are working at CAE teams of big companies showing their expertise on FEM. But soon they should demonstrate another capability of creativity when FEM becomes a routine engineering task. So far there is a specialty called 'numerical analyst', but the name will soon disappear. That is also the reason that students of engineering science should learn TRIZ more seriously. The ideal final CAE systems will free engineers from solutions of difficult differential equations and long hours to master FEM proficiently. By then the engineers can concentrate only on creative and innovative idea generation [12]. 
Engineering design can be divided into formative and creative design. The formative design is incremental design, routine design, improvement, optimization, and compromise. Creative design is innovative design that aims to implement an original concept beyond current paradigm. Even though the importance of creative design is recognized, there are no other methods than TRIZ to enhance systematic creativity in real world design problems.

\section{Implications on Engineering Education}

\subsection{New required capabilities of engineers in global design environment}

For an engineering educator, the required skills which are needed as global engineers are the most important concern. Hayes and Pande identified 5 trends in the global market place as more internationally distributed organizations, more distributed product development teams, more complex products, more products aiming at emerging markets, and products needed to be more usable and appealing [13]. They also summarized 5 more skills in addition to technical expertise for effective global engineers including cross-cultural skills, systems thinking skills, willingness to work outside one's own discipline, design for usability skills, and new approaches to design management. As a structural mechanics professor studying CAE mainly on FEM, I feel that we should add creativity to achieve those goals. Next section will examine this idea.

\subsection{Systematic Innovation}

One of the big concerns of practicing engineers is that these capabilities do not guarantee a commercial success of an engineering product. Some technological breakthroughs are required in addition to fancy colorful drafts of analysis results and designs. So a new paradigm for the product development is sought and CAI (Computer Aided Innovation) can be the answer for that requirement. Naturally CAI, such as TRIZ softwares are rapidly gaining momentum for practical applications. As confessed in the article of ASEE (American Society of Engineering Education) magazine, PRISM, the engineering education community couldn't find good tools for creativity except serendipity [14]. Only luck and probability dominates in this much laborious approach. The article pointed out TRIZ as the potential solution for creativity in engineering education. Prof. Flowers of MIT stressed that creativity is so important that 'informed creative thinking' must be taught in engineering education in his plenary talk at the 2004 ASEE conference [15]. The reason is that smart students can be obtained by outsourcing but the leadership can not be outsourced. In addition to that point he declared engineering science is dead and real engineering is needed in engineering education. There are many debates on what creativity is and how to foster creativity. Researches on creativity can be divided into 3 categories, namely on creative people, methods and products. One Japanese 
scholar summarized 88 methods of creativity [16]. But the important thing is how to make people learn and improve their creative activities. So the systematic innovation tools are long sought and developments into softwares are now in the realm of CAI. We spent a long time to investigate these tools and found TRIZ could be much helpful for our education and also for generating new products and patents.

\subsection{CAI in Engineering Education}

In our school, TRIZ has been introduced to the class for 7 years in the courses of Creative Problem Solving (CPS) for freshman, Creative Engineering Design (CED) for junior and New Product Design (NPD) for senior. Traditional engineering design texts have been used for many years before TRIZ since Engineering Design courses have been a mandatory course. Recent Korean texts are being used for design education $[17,18]$. The first year engineering students practice engineering process of design using LEGO system and the concept of creative practice is just explained. At the junior course, a more serious process is adopted. Teams are made of 4 students to make as diverse as possible. They are assigned 2 projects during the semester. The first is targeted to bring 'Eyes for Design'. They should make a long list of items that they feel represent bad or uncomfortable design. This part is 'Need Finding' stage, which may be considered as same as QFD in product development. Next project is to improve or solve that situation. Usual outcomes from this course for many years before introduction of TRIZ were CAD drawings. At most better reports included the FE analysis results. Virtually no outcomes were considered to have serious practical meaning. After the introduction of TRIZ, the results became several applications of patents and designs at the end of semester. Some of them were developed into more serious government funded projects. TRIZ contributed by motivating the students to follow all of the steps of patent applications and also give confidence in the sense of what they can do. From last year, we started to offer senior NPD course by popular requests from students. Here we directly apply TRIZ principles and processes for given subject by using 3 commercial TRIZ softwares, namely Goldfire, Innovation WorkBench, and CREAX. Their responses and the outcomes are great. Every team prepared patents and/or business plan for their products. And they made a study group of TRIZ and became evangelists among students. I have not seen such enthusiastic responses and practical outputs before. This fact gives a big momentum for Korean universities and there is a movement to form a coalition for creative engineering design adopting this approach. This can be a manifest of the trend 'from engineering science to engineering'. It also gives 2 implications to engineering education. As the required credits for a degree become smaller in current college of engineering curricula, the material of the basic science courses should be compacted and more courses of exercising creativity should be developed. In the light of this trend, the purpose of engineering education involves from training to adapt current technology to breeding self-learning capabilities. We found the approach of 'Effects' module in Goldfire is very promising. They collected 8000 important scientific principles and categorized by verbs applied to fields, parameters and substances. This taxonomy of engineering activities can be more elaborated and transferred to engineering students efficiently. One of the important topics in solid mechanics 
which is one of the core courses in mechanical engineering is to analyze a cantilever beam. Only few students recognize the usage of a cantilever beam as a sensor on the airbag until they were asked about the usage. Instead of extending 1-d mechanics to bending problem, we may ask them to find ways to measure the acceleration. As this kind of extension has big potential for engineering education, we organized a multiuniversity research team and started a project to elaborate the taxonomy of engineering activities. This approach is expected not only to boost student's problem solving capabilities overcoming narrow sight in a single core discipline but also to give practically more freedom to concentrate on the essence of problems instead of on the methods themselves. These efforts are applied in diverse ways of teaching to utilize current IT advancements. Distance learning and certification of TRIZ is being tried for reeducation of practicing engineers. This system is brought into AUCSE (Ajou University Consortium for Staff Enrichment) which was built in 2006 to educate the people and solve the problems of small and medium sized companies. The most widely sought topics from survey were some depth technology of their own and the capabilities to produce successful new product. More elaborate study on the education system of these approaches is expected to change current engineering education system.

\section{Conclusions}

The vision of next-generation engineers may be described as follows. Every engineer with capability of simulating whatever they want to do using computer and IT technology is concentrating on his mission and solve by exercising his creativity by help from CAI softwares. To this end, we have examined the history of FEM and predicted the future of CAE. The necessity of creativity came out naturally from this investigation and our experiences of implementing TRIZ into our design classes are described. The efforts to renovate current engineering education systems are also introduced. All attempts to collaborate in this direction and globalize engineering education are welcomed. And this is the point where we should show our learned creativity and exercise CAI capabilities.

\section{References}

1. L. Leifer, et. al., Radical Innovation: How Mature Companies Can Outsmart Upstarts (Harvard Business School Press, 2000).

2. M. Stefik, and B. Stefik, Breakthrough : Stories and Strategies of Radical Innovation (The MIT Press, 2004)

3. W. Sun, Strategy to Connect Manpower of Industries and Universities, Mechanical Engineer's Day Conference, KSME (2004)

4. CAIB (Columbia Accident Investigation Board) report, USA http://caib.nasa.gov (2003)

5. O.C. Zienckiwicz, R.L. Taylor, and J.Z.N. Zhu, The Finite Element Method, (Butterworth-Heinemann, $6^{\text {th }}$ ed., 2005) 
6. C. F. Williamson, Jr., A History of the Finite Element Method to the Middle of 1960's, Ph. D. Thesis (Boston University, 1976)

7. Homepage of the TRIZ journal, http://www.triz-journal.com/

8. P. Bettess, and O.C. Zienckiwicz, Diffraction and Refraction of Surface Waves Using Finite and Infinite Elements, Int. J. Numer. Meth. Eng. 11, 1271-1296 (1977)

9. M. Bryden, Virtual Engineering : Playing the Real Game, Seminar at Univ. of Maryland (2007)

10. J.T. Oden, et. al., Research Directions in Computational Mechanics, Comp. Meth. in Appl. Mech. and Eng., 192, 913-922 (2003)

11. World Community Grid Hompage : http://www.worldcommunitygrid.org/

12. N. Leon, and O. Martinez, Product Optimization vs. Innovation, Steps toward a Computer Aided Inventing Environment, Proc. TRIZCON 2003, Philadelphia, PA, 2-1 2-12 (2003)

13. C. C. Hayes, and A. Pande, What Skills Will Future Engineering Graduates Need in Global Organizations?, Proc. Int. Design Eng. Tech. Conf., ASME (2007)

14. D. McGraw, Expanding the Mind, PRISM, ASEE, 13(9), 30-36 (2004)

15. W. Flowers, Plenary Talk, ASEE Annual Conference, June 27 (2004)

16. T. Makoto, The Bible of Creativity (Moodo School Press, Japan, 1993) (In Japanese)

17. S.H. Yoo, Designer's Creativity, (Ajou University Press, Suwon, Korea, 2004) (In Korean)

18. I.C. Kim, What to make?, (Intervision, Korea 2006) (In Korean) 